\title{
Strategies for Next Generation Neutrinoless Double-Beta Decay Experiments
}

\author{
F. T. Avignone III \\ Department of Physics and Astronomy, University of South Carolina, Columbia, South Carolina 29208, \\ USA
}

A brief discussion of the connection between neutrino oscillation data and predictions of neutrinoless doublebeta decay half-lives is given. Two convenient figure of merit formulae are discussed in the context of the various isotopes and technologies proposed. These allow definite evaluation of the merits of the various proposed experiments. Other criteria are discussed, which in total, if applied, should give all the necessary tools for comparative evaluation.

\section{INTRODUCTION}

Neutrinoless double-beta $(0 \nu \beta \beta)$-decay has been reviewed in detail many times in the literature [1-8], and discussed in these proceedings. The positive observation of atmosphericneutrino oscillations [9], and solar-neutrino oscillations [10], and the measurement of their parameters, allows the prediction of the range of estimated half-lives of $0 \nu \beta \beta$-decay candidates if neutrinos are Majorana particles. The ranges of mixing angles and neutrino eigenstate masses derived from oscillation data are consistent with values of the effective Majorana mass of the electron neutrino, $\left\langle m_{\nu}\right\rangle$, corresponding to half-lives of some candidate $0 \nu \beta \beta$-decay isotopes within reach of several of the proposed next generation experiments [11-13]. The mean life of this exotic process, when driven by Majorana neutrino exchange, is related to $\left\langle m_{\nu}\right\rangle$ as follows:

$$
\left(T_{1 / 2}\right)^{-1}=\frac{\left\langle m_{\nu}\right\rangle^{2}}{\left\langle m_{e}\right\rangle^{2}} G^{0 \nu}\left|M^{0 \nu}\right|^{2},
$$

where $G^{0 \nu}$ is the phase space factor, including coupling constants, and $M^{0 \nu}$ is the nuclear matrix element of the decay. The connection between the neutrino mixing matrix and $\left\langle m_{\nu}\right\rangle$ is by now well known:

$\left|\left\langle m_{\nu}\right\rangle\right|=\left.|| U_{e 1}^{L}\right|^{2} m_{1}+\left|U_{e 2}^{L}\right|^{2} m_{2} e^{i \varphi_{2}}+\left|U_{e 3}^{L}\right|^{2} m_{3} e^{i\left(\varphi_{3}-\delta\right)} \mid$,

where $U_{e_{k}}$ are the elements of the first row of the mixing matrix, $m_{k}$ are the three neutrino mass eigenvalues, and $e^{i \varphi_{2,3}}$ are Majorana $\mathrm{CP}$ phases, with $e^{i \delta}$ being the $\mathrm{CP}$ phase that appears in the quark mixing matrix. The Majorana phases do not appear in neutrino oscillation expressions; $e^{-e \delta}$ does.

The following approximate equations can be written by neglecting $\left|U_{e_{3}}\right|$ which is much smaller than both $\left|U_{e_{1}}\right|$ and $\left|U_{e_{2}}\right|$. The known relationships between the three neutrino mass eigenstates allow the approximate equations to be written in terms of $m_{1}, \delta m_{A T}^{2}$ and $\delta m_{\text {solar }}^{2}$ as follows [13]:

$$
\left.\left\langle m_{\nu}\right\rangle \simeq m_{1}|| U_{e 1}^{L}\right|^{2}+\left|U_{e 2}^{L}\right|^{2} e^{i \varphi_{2}}\left(1+\frac{\delta m_{\text {solar }}^{2}}{2 m_{1}}\right)
$$

in the case of normal hierarchy: $m_{1} \simeq m_{2} \ll m_{3}$, and

$$
\left.\left\langle m_{\nu}\right\rangle \simeq \sqrt{m_{1}^{2}+\delta m_{A T}^{2}}|| U_{e 1}^{L}\right|^{2} e^{i \varphi_{2}}+\left|U_{e 2}^{L}\right|^{2} e^{i \varphi_{3}} \mid,(4)
$$

in the case of inverted hierarchy: $m_{1} \ll m_{2} \simeq m_{3}$. In these approximations $\left|U_{e 1}^{L}\right|^{2}=\cos ^{2} \theta_{12} \simeq 0.70$, and $\left|U_{e 2}^{L}\right|^{2}=\sin ^{2} \theta_{12} \simeq 0.30$.

From equation (4), we conclude that real next generation $0 \nu \beta \beta$-decay experiments must at least have the sensitivity to probe the coherent case of the inverted hierarchy $\left(e^{i \varphi_{2}}=e^{i \varphi_{3}}\right)$, when $m_{1} \simeq 0$. Accordingly, $\left\langle m_{1}\right\rangle \simeq \sqrt{\delta m_{A T}^{2}} \approx(40-$ 50) $\mathrm{meV}$.

In the later sections, the available isotopes and technologies are discussed. The important figure of merit formulae are discussed that should 
be considered when evaluating the relative merits of the various proposals. They emphasize the importance of isotopic abundance of the source, detection efficiency, energy resolution, and background rejection. The total mass of the source is obviously very important.

At this point it is convenient to introduce the nuclear factor $F_{N} \equiv G^{o \nu}\left|M^{0 \nu}\right|^{2}$, such that $\left\langle m_{\nu}\right\rangle=m_{e} / \sqrt{F_{N} T_{1 / 2}}$. The average values taken over many reported calculated values are used in our estimates, and for convenience we define $\bar{\eta} \equiv\left\langle F_{N}\right\rangle \times 10^{13} y^{-1}$. We will use this parameter frequently later.

\section{AVAILABLE ISOTOPES}

There are more than 30 isotopes with the eveneven pairing energy level structure that forbids weak decay to the next odd-odd nucleus because it would not conserve energy. In Table 1, we list the 8 that have large decay energies and for which there are methods of isotopic enrichment. These are the ones that are most frequently the subjects of experimental proposals.

In the second column, values of the average nuclear structure factor appear. There is no real justification for depending on the absolute value of these as being accurate. They are the average values mainly taken from the list given by Tretyak and Zdesenko [14], and work published since. A few calculations that were shown later to be incorrect were not included. Also not included were earlier calculations of authors who published results later using the same general approach, and in cases in which it is clear that the authors imply that their later work should replace their earlier one. The uncertainty quoted is the $1 \sigma$ mean square deviation. These values are only meant to project the general centroid of the theoretical rates. Though the absolute values can not be considered highly reliable, they do represent the best estimates we have of the predicted decay rates. The parameter, $\bar{\eta}$, is useful in comparing the relative merits of proposals and should be included in the comparative analysis. While not claimed to be highly accurate, it is clear that we should expect the decay rate of ${ }^{100} M o(\bar{\eta} \simeq 5.0)$, for example, to be significantly larger than that of ${ }^{136} \mathrm{Xe}(\bar{\eta} \simeq 0.28)$. This fact is an important consideration.

We should not take the value of $\bar{\eta}\left({ }^{150} N d\right)$ as seriously as the others because there are only very few calculations. In addition the very large static quadrupole moment of the ground-state makes this calculation extremely difficult. This technique of averaging many published theoretical results will suffer criticism; however, it does give us general estimates of the relative expected decay rates.

\section{FIGURE OF MERIT FORMULAE}

The general criterion for discovery can be expressed as $C_{\beta \beta}=C_{1} \sqrt{B+C_{\beta \beta}}$, where $C_{\beta \beta}$ is the number of $0 \nu \beta \beta$-decay events, $B$ is the background in the region of interest (ROI), and $C_{1}$ is related to the confidence level in units of $\sigma$ of the Poisson distribution of the spectrum in the ROI. For simplicity we can require some specific signal-to-background ratio, for example $C_{\beta \beta} / B \simeq$ 1. In this case we require $C_{\beta \beta}=\gamma \sqrt{B}$ where $\gamma=C_{1} \sqrt{2}$. This choice is arbitrary, however, it can be made the same for all experiments being compared.

The criterion above can be written as follows:

$C_{\beta \beta}=\lambda_{\beta \beta} N t \epsilon=\gamma \sqrt{b M t \delta E}$,

where $\lambda_{\beta \beta}=\tau_{\beta \beta}^{-1}$, the decay rate, $N$ is the number of parent nuclei and $\epsilon$ is the detection efficiency. On the right hand side $\gamma$ is defined above, $b$ is the background rate in counts $k g^{-1} \mathrm{keV}^{-1} \mathrm{y}^{-1}, t$ is the operating time, $\delta E$ is the optimum window of the analysis for a search for the $0 \nu \beta \beta$-decay peak, $M$ is the mass of the source in $\mathrm{kg}$, and $\delta E$ is proportional to the energy resolution. We can replace it by the full width at half maximum (fwhm) of the experiment.

From this it is easy to show that the half-life sensitivity can be written as follows:

$T_{1 / 2}^{0 \nu}=\left(\frac{A_{0} \times 10^{3}}{\gamma}\right)\left(\frac{a \epsilon}{W}\right) \sqrt{\frac{M t}{b \delta E}}$.

In equation (6) $A_{0}$ is Avagadro's number, $a$ is the isotopic abundance, and $W$ is the molecular weight of the source. 
The parameter, $\bar{\eta}$, discussed earlier (see Table 1 ), is proportional to $\lambda_{\beta \beta}$, and hence inversely proportional to the theoretical prediction of the half-life for some arbitrary choice of the parameter $\left\langle m_{\nu}\right\rangle$.

A very comprehensive expression for the relative figure of merit is one that is proportional to ratio of the half-life sensitivity of a proposed experiment under consideration and the theoretical half-life which, for the choice of $\left\langle m_{\nu}\right\rangle$, is proportional to $\bar{\eta}^{-1}$. This figure of merit is expressed as follows:

$f=\bar{\eta}\left(\frac{a \epsilon}{W}\right) \sqrt{\frac{M}{b \delta E}}$,

where we have eliminated parameters common to all experiments being compared.

There is another rather unconventional figure of merit that places more emphasis on the properties of an experiment that enhances our ability to find the "needle" of signal in the "haystack" of background. This is the ratio of the expected signal in the "haystack" of background. This is the ratio of the expected signal for a target value of $\left\langle m_{\nu}\right\rangle$, to the expected background. This is simply:

$f_{d} \equiv \frac{\lambda_{\beta \beta} N t \epsilon}{b M \delta E t} \propto \frac{\bar{\eta} a \epsilon}{W b \delta E}$.

While very useful in comparing some experiments, it is not very useful in comparing those vastly different in source mass. In cases of zero background, and adequate energy resolution to exclude interference from $2 \nu \beta \beta$-decay, equation (8) will not be useful.

\section{SAMPLE APPLICATIONS}

\subsection{Germanium experiments}

There are four proposed ${ }^{76} \mathrm{Ge}$ experiments: GEM [15], GENIUS [16], Majorana [17] and the new ${ }^{76} \mathrm{Ge}$ experiment proposed for Gran Sasso [18]. Let us use as an example, the well documented parameters from the Majorana White paper [17]. In this case: $a=0.86, \epsilon=0.75, W=$ $76, M=500 \mathrm{~kg}$, and $\delta E=3.5 \mathrm{keV}$. From Table $1, \bar{\eta}=0.73$, and from Table $2-4$ of [17] we deduce $b=0.005 \mathrm{keV}^{-1} \mathrm{~kg}^{-1} \mathrm{y}^{-1}$, without using the segmentation cut, which is unique to Majorana. Accordingly, $f=1.15$ for a typical well executed ${ }^{76} \mathrm{Ge}$ experiment. This is conservative since most proposals claim target values of $b<0.005 \mathrm{keV}^{-1} \mathrm{~kg}^{-1} \mathrm{y}^{-1}$. We will then use $f \simeq 1.0$ as the canonical value of, $f$, that defines a highly competitive proposed experiment.

For the values of the parameters above, $f_{d}=$ 0.344 . For convenience, we multiply equation (8) by 2.91 so that the canonical value of, $f_{d}$, is also 1.0. Accordingly we use,

$f_{d}=2.91 \bar{\eta} a \epsilon / W b \delta E$.

\subsection{Cryogenic experiments}

Next we consider the CUORE proposal which is the only example of a cryogenic $0 \nu \beta \beta$-decay experiment. It is the only other proposal with excellent energy resolution, $\delta E \simeq 7 k e v$ and it also has a successful pilot experiment, CUORICINO, to document its performance.

First, consider $\mathrm{TeO}_{2}$ bolometers with natural abundance, $33.8 \%$. In this case $a=0.338, \epsilon=$ $0.84, W=163, M=760 \mathrm{~kg}, b=0.005$, and $\delta E=7 \mathrm{keV}$. In this case $f=1.09$, while $f_{d} \simeq 0.62$. While comparable to Majorana, the main differences are from the isotopic abundance and the energy resolution. If CUORE was isotopically enriched, $F=2.76$, and $f_{d}=1.55$. These optimistic figures depend on achieving the factor of 40 background reduction over the background in CUORICINO, $0.2 \mathrm{keV}^{-1} \mathrm{~kg}^{-1} \mathrm{y}^{-1}$. According to the talk by my colleague Ettore Fiorini (see E. Fiorini these proceedings), significant progress is being made towards this goal.

\subsection{Large Xe experiments}

There are three large xenon-136 proposals, EXO [19], XMASS [20], and Xe-Borexino [21]. In the case of EXO, there is an intense R \& D effort to reduce the background to essentially zero by capturing the ${ }^{136} \mathrm{Ba}^{+}$ion, after one charge neutralization of ${ }^{136} \mathrm{Ba}^{++}$, bringing it to the intersection of two lasers and using resonant photoexcitation and decay of the $6^{2} P_{1 / 2}$ atomic state at $493 \mathrm{~nm}$ which decays to a $5^{4} D_{3 / 2}$ metastable state. A second laser, $650 \mathrm{~nm}$ excites it back to the $6^{2} P_{1 / 2}$ level and as many as $10^{7}$ photons $\mathrm{sec}^{-1}$ are emitted which can be used to identify 
the ${ }^{136} \mathrm{Ba}^{+}$daughter nucleus. This follows the original suggestion of Moe [22]. This has not yet been achieved, and it presents a significant challenge.

For purposes of discussion, let us estimate, f, for a 10 ton ${ }^{136} \mathrm{Xe}$ time projection chamber with an estimated overall efficiency $\epsilon=0.3, a=0.8$ and $\delta E=50 \mathrm{keV}$, which has been achieved in a small chamber using charge collection and scintillation light. In this case we can express the figure of merit, $f$, in terms of $b^{-1 / 2}$ as follows:

$f=0.007 b^{-1 / 2}$.

The required background level would be $b \sim$ $5 \times 10^{-5} \mathrm{keV}^{-1} \mathrm{~kg}^{-1} \mathrm{y}^{-1}$, to reach $f=1$. This is the challenge of the EXO collaboration to achieve success with the ${ }^{136} \mathrm{Ba}^{+}$tagging technique. One very uncertain parameter is the efficiency, $\epsilon$, associated with the tagging. For a one ton Xe TPC experiment, the required background level is $\sim 5 \times 10^{-6} \mathrm{keV}^{-1} \mathrm{~kg}^{-1} \mathrm{y}^{-1}$.

If we consider the parameters given in [20] for the XMASS experiment, and assume $a=$ 0.80 , with a photo-cathode coverage of $70 \%$, we conclude that the resolution of the $100 \mathrm{~kg}$ version $(\delta E=580 \mathrm{keV})$ could be reduced to $\delta E \simeq 133 \mathrm{keV}$ in the $800 \mathrm{~kg}$ version. Using these estimates $f \simeq 0.03$. If the background could be reduced to $10^{-3} \mathrm{keV}^{-1} \mathrm{~kg}^{-1} \mathrm{y}^{-1}$ then $f \simeq 0.1$. To achieve a figure of merit of unity, the background rate will have to be reduced to $b \simeq 10^{-5} \mathrm{keV}^{-1} \mathrm{~kg}^{-1} \mathrm{y}^{-1}$, a significant challenge.

The third large ${ }^{136} \mathrm{Xe}$ proposal, Xe-Borexino [21] proposes an experiment in which $1565 \mathrm{~kg}$ of $\mathrm{Xe}$, enriched to $80 \%{ }^{136} \mathrm{Xe}$ is dissolved in the Borexino scintillator. The following parameters were obtained from [21]: $a=0.8, \epsilon=$ $0.68, \quad M=1565 \mathrm{~kg}, \delta E=186 \mathrm{keV}, \quad b=$ $3 \times 10^{-4} \mathrm{keV}^{-1} \mathrm{~kg}^{-1} \mathrm{y}^{-1}$. Using the parameters $\bar{\eta}=0.28$, and $W=136$, we obtain the value $f \simeq 0.19$. To reach $f \simeq$ unity, the background rate must be reduced to $b \simeq 10^{-5} \mathrm{keV}^{-1} \mathrm{~kg}^{-1} \mathrm{y}^{-1}$.

To put these incredibly low background rates in perspective, we revisit the projected rate of the Majorana experiment over $2500 \mathrm{~kg} . \mathrm{y}$. With the application of pulse shape discrimination and detector segmentation, the calculated result is that 7 background counts will remain. This corre- sponds to $b=2.8 \times 10^{-3} \mathrm{keV}^{-1} \mathrm{~kg}^{-1} \mathrm{y}^{-1}$. To exceed this value by one or two orders of magnitude will be a real challenge.

\subsection{The DCBA ${ }^{150} N d$ experiment}

The Drift Chamber beta-ray analyzes (DCBA) is the only serious effort underway at this time to exploit the large value of $\bar{\eta}$ of ${ }^{150} \mathrm{Nd}$ [23]. There is not enough known about the parameters of DCBA to confidently compute the figure of merit. What is known are $\delta E \simeq 200 \mathrm{keV}$ and $N\left({ }^{150} N d\right) \simeq 7.5 \times 10^{25}$ atoms. According to Table 2, an experiment will have to reach the sensitivity of $T_{1 / 2}^{0 \nu} \simeq 2.9 \times 10^{25}$ years to probe the sensitivity $\left\langle m_{\nu}\right\rangle \simeq 0.04 \mathrm{eV}$. If we estimate a counting efficiency of $\epsilon \simeq 0.5$, the experiment will have to yield less than 1 count/year in background. If the $\mathrm{Nd}$ is in the form of $\mathrm{Nd}_{2} \mathrm{O}_{3}$, then $\mathrm{W}=348$. We then compute that $M=28.3 \mathrm{~kg}$ of $\mathrm{Nd}_{2} \mathrm{O}_{3}$ if $a \simeq 0.8$. Using these parameters we see $b \simeq 0.035 \mathrm{keV}^{-1} \mathrm{~kg}^{-1} \mathrm{y}^{-1}$. From Table 1, $\bar{\eta} \simeq 57$. With this we arrive at $f=0.25$.

This seems respectable; however, the large value $\delta E \simeq 200 \mathrm{keV}$ very probably lead to difficulties with the contamination of the ROI at $\left|Q_{\beta \beta}\right|$ with counts from $2 \nu \beta \beta$-decay. In addition, the discovery potential indicator of equation (10) yields $f_{d} \simeq 0.05$. This should be intuitively obvious if we assume that $\left\langle m_{\nu}\right\rangle \simeq 0.04 \mathrm{eV}$, we will observe $\lambda_{\beta \beta} N t \epsilon \simeq\left(2.4 \times 10^{-26} y^{-1}\right)(4.16 \times$ $\left.10^{25}{ }^{150} N d\right)(\epsilon \simeq 1.0) / y \simeq 1 y^{-1}$ with this apparatus. Spread over $200 \mathrm{keV}$ (fwhm), a discovery at $\left\langle m_{\nu}\right\rangle \simeq 0.04$ will not be possible. This example points out the weakness of relying two heavily just an figure of merit formulae. The total mass and count rates, $\lambda_{\beta \beta} N \epsilon$, must also be considered.

\subsection{Scintillator experiments}

CAMEO. This proposal involves $100 \mathrm{~kg}$ of ${ }^{116} \mathrm{Cd} \mathrm{WO}_{4}$ scintillation detectors to investigate the $0 \nu \beta \beta$-decay of ${ }^{116} C d[24]$. The plan is to place these detectors in a high purity liquid in the Boreximo Counting Test Facility. The projected parameters are: $\bar{\eta}=1.3, \epsilon=1, a=$ $0.83, \delta E=112 \mathrm{keV}, M=100 \mathrm{~kg}, W=360$, and $b=0.0004 \mathrm{keV}^{-1} \mathrm{~kg}^{-1} \mathrm{y}^{-1}$. This background is a factor of 100 lower than that already reached in the Solotvina laboratory [25]. With these tar- 
get parameters, we see $f=0.14$.

The number of ${ }^{116} \mathrm{Cd}$ atoms in $100 \mathrm{~kg}$ of $\mathrm{CdWO}_{4}$ with $83 \%$ enriched ${ }^{116} \mathrm{Cd}$, is $1.39 \times 10^{26}$. The predicted half-life corresponding to $\left\langle m_{\nu}\right\rangle \simeq$ $0.04 \mathrm{eV}$ is $1.3 \times 10^{26}$. The predicted decay rate, $\lambda \beta \beta N \epsilon=0.07 y^{-1}$. Again, this experiment will need significant expansion to be competitive, even though $f_{d}=0.0007 \div 0.0004=1.75$.

Again, one can call into question the estimated average value, $\bar{\eta}$, from Table 1 . Nevertheless, even if it is off by a factor of 10 , the experiment would need significantly more mass.

If one refers to Table 3 , one can easily determine from the column labelled, $N \lambda_{\beta \beta}$, the mass required to make a statistically significant discovery at $\left\langle m_{\nu}\right\rangle \simeq 0.04 \mathrm{eV}$. If one takes a different approach and starts with Table 3, it might be immediately clear that $100 \mathrm{~kg}$ of $\mathrm{Nd}_{2} \mathrm{O}_{3}$ enriched to $85 \%$ is roughly equivalent to $10^{6} \mathrm{~kg}$ of $\mathrm{CaF}_{2}$ with natural abundance $\mathrm{Ca}$. It becomes then clear that $5000 \mathrm{~kg}$ years of ${ }^{76} \mathrm{Ge}$ detector operation might have a respectable discovery potential with $\sim 10$ events in a narrow energy region $(\sim 3.5 \mathrm{keV})$ but if and only if the background is roughly the same or less, i. e., $(2-4) \times 10^{-3} \mathrm{keV}^{-1} \mathrm{~kg}^{-1} \mathrm{y}^{-1}$.

If on the other hand, one chooses to use ${ }^{136} X e$ in a TPC, one needs $2.3 \times 10^{4} \mathrm{~kg} \cdot y$ to obtain 10 events at $\left\langle m_{\nu}\right\rangle \simeq 0.04 \mathrm{eV}$. These events will be spread over $\sim 50 \mathrm{keV}$ in the most optimistic case. So it will be necessary to reduce the background to nearly zero. This is of course the intention of the EXO collaboration and the motivation of the $\mathrm{R} \& \mathrm{D}$ program to tag the ${ }^{136} B \mathrm{a}^{+}$ion.

\subsection{Metal foil-scintillator tracking cham- bers}

The MOON (Molybdenum Observatory Of Neutrinos) experiment is a proposed series of molybdenum foils sandwiched between plastic scintillators [25]. There is not enough data on the detection efficiency or background to calculate an accurate figure of merit; however, the following parameters are reasonable: $a=0.9, \bar{\eta}=$ $5.0, W=100, M=1000 \mathrm{~kg}$, and, $\delta E=5.4 \% \cong$ $190 \mathrm{keV}$. Let us assume an efficiency $\varepsilon=0.5$. Accordingly, for $f=1.0$,

$\frac{\bar{\eta} a \epsilon}{W} \sqrt{\frac{M}{\delta E}}=\sqrt{b}$.
In this case a background of 0.014 counts $\mathrm{keV}^{-1} \mathrm{~kg}^{-1} \mathrm{y}^{-1}$ would result in a figure of merit of 1.0. This will be a significant challenge, but possibly achievable. Reference to Table 3 , however, gives one a good qualitative feel for the discovery potential. It predicts $\sim 110 \nu \beta \beta$-decay counts per $1000 \mathrm{~kg}$ of $90 \%{ }^{100} \mathrm{Mo}$ per year. It would require roughly 10 ton years to observe $\sim 110$ events over $\sim 200 \mathrm{keV}$. To maintain a signal to background ratio of $\sim 1$, the background rate will have to be restricted to $\sim 5.5 \times 10^{-5} \mathrm{keV}^{-1} \mathrm{~kg}^{-1} \mathrm{y}^{-1}$. Again we see the importance of energy resolution for discovery potential. A molybdenum detector with $<10 \mathrm{keV} \mathrm{fwhm}$ would be a powerful detector, a bolometer perhaps.

\section{CONCLUSIONS}

It is not necessary to continue the exercise of subjecting all of the various proposals to the criteria suggested here. The main points should now be clear. The figure of merit formulae discussed earlier contain a necessary factor proportional to the estimated $0 \nu \beta \beta$-decay rate for a given effective mass of the electron neutrino. This is usually not included, but is absolutely necessary.

In subjecting the various experimental proposals to same litmus test, equations (7) and (8) can give general guidance of a relative merit. This should be followed by consulting Table 3 from which one can conclude how many $\mathrm{kg}$. years of data would be necessary to make a discovery if neutrinos are Majorana particles, and if the eigenvalue mass spectrum is inverted (i. e., $\left.\left\langle m_{\nu}\right\rangle \sim 0.04 \mathrm{eV}\right)$.

To make a final comparison of experimental proposals concerning discovery potential, it is necessary to consider both experimental energy resolution and background. The important questions are - how many real $0 \nu \beta \beta$-decay events are expected in a reasonable time, and how probable is it that the "needle of real events" can be discovered in the "haystack of background".

Finally, the organizers have asked me to state which of the many proposals I would favor. To them I say, thank you for inviting me to speak to the neutrino community. To answer your question, don't just listen to what I say, watch what I 
do. I have chosen my two favorite experiments to devote my energies to. They both have the best of the parameters we have been discussing, and in particular, sharp energy resolution.

\section{REFERENCES}

1. H. Primakoff and S.P. Rosen, Phys. Rev. 184 (1969) 1925.

2. W.C. Haxton and G.J. Stevanson, Jr., Progress in Particle and Nuclear Physics.

3. M. Moe and P. Vogel, Ann. Rev. Nucl. Part. Sci. 44 (1994) 247.

4. F.T. Avignone III, and R.L. Brodzinski, Prog. in Part. and Nucl. Phys. 21 ( ) 99.

5. S.R. Elliott and P. Vogel, Ann. Rev. Part. Sci. 52 (2002) 115.

6. S.R. Elliott and J. Engel, hep-ph/0405078, submitted to J. Phys. G.

7. H. Primakoff and S.P. Rosen, Ann. Rev. Nucl. Part. Sci. 31 (1981) 145.

8. Yu.G. Zdesenko, Rev. Mod. Phys. 74 (2002) 663.

9. S. Fakuda et al., The Super Kamiokande Collaboration, Phys. Lett. B 539 (2002) 179 (see references therin).

10. Q.R. Ahmed et al., The SNO Collaboration, Phys. Rev. Lett. 87 (2001) 071301; Phys. Rev. Lett. 92 (2004) 181301 (also see references therin for earlier work).

11. J.N. Bahcall, M.C. Gonzales-Garcia, and C. Peña-Garay, ArXiV:hep-ph/0406294; This 31 page article gives a complete stay of the past present and future of the subject.

12. S. Pascoli and S.T. Petcov, Phys. Lett. B 544 (2002) 239.

13. "The Neutrino Mixing Matrix, Neutrino Oscillations, and Neutrinoless Double-Beta Decay", F.T. Avignone III and G.S. King III, Proc. $4^{\text {th }}$ Workshop on the Identification of Dark Matter, York, UK, 2-6 September 2002, eds. Neil J.C. Spooner and Vitaly Kudryavtsev, World Scientific pp. 553 (2003).

14. I.V.Tretyak and Yu.G. Zdesenko, At. Data Nucl. Data Tables 80 (2002) 83.

15. Yu.G. Zdesenko, O.A. Ponkratenko, and V.I. Tretyak, J. Phys. G: Nucl. Part. Phys. 27 (2001) 2129.
16. H.V. Klapdor-Kleingrothaus, J. Hellmig, and M. Hirsch, J. Phys. G 24 (1998) 483; see also the complete white paper, H.V. KlapdorKleingrothaus et al., hep-ph/9910205 v4 June 29, 2000.

17. "The Majorana Zero-Neutrino Double-Beta Decay Experiment," LA-UR-2003-7709, and PNL-1440, Nov. 3, 2003: http: //majorana.pnl.gov.

18. I. Abt et all., "A New ${ }^{76} G e$ Double-Beta Decay Ecperiment at LNGS," MPT Letter of Intent, arXiV: hep-ex//0406035 v1 13 June, 2004.

19. M. Danilow et all., Phys. Lett. B 480 (2000) 12.

20. S. Moriuama (the XMASS Collaboration) Proc. Int. Workshop on Techniques and Applications of Xenon Setectors, Tokyo, Japan, 3-4 Dec. 2001, World Scientific, Singapure (2002) p. 123; see also http://www-3k.icrr.Utokyo.ac.jp/ minamino/doc/KEKPH_Mar_2004.pdf.

21. B. Caccianiga and M.G. Giammarchi, Astroparicle Physics 14 (2000) 15.

22. M.K. Moe, Phys. Rev. C 44 (1991) R 981.

23. N. Ishihara et all., Nucl. Instrum. and Meth. A 433 (2001) 101; Y. Kato et all., Nucl. Instrum. and Meth. A 498 (2003) 430.

24. G. Bellini et all., Eur. Phys. J C 19 (2001) 43; Phys. Lett. B 493 (2000) 216.

25. H. Ejiri et all., Phys. Rev. C 63 (2000) 065501. 
Table 1

The most popular parent isotopes, their method of isotopic enrichment, average nuclear structure factor, $\left\langle F_{N}\right\rangle \equiv\left\langle G^{0 \nu}\left|M^{0 \nu}\right|^{2}\right\rangle y^{-1}$, the renormalized nuclear structure factor $\bar{\eta} \equiv\left\langle F_{N}\right\rangle \times 10^{13} y^{-1}$, and total $0 \nu \beta \beta$-decay energy $\left|Q_{\beta \beta}\right|$ in $\mathrm{keV}$.

\begin{tabular}{|l|l|c|c|}
\hline \hline Parent Isotope & $\left\langle F_{N}\right\rangle \equiv\left\langle G^{0 \nu}\left|M^{0 \nu}\right|^{2}\right\rangle y^{-1}$ & $\bar{\eta} \equiv\left\langle F_{N}\right\rangle \times 10^{13} y^{-1}$ & $\left|Q_{\beta \beta}\right| k e V$ \\
\hline${ }^{48} \mathrm{Ca}(\text { Cent. })^{\dagger}$ & $\left(5.4_{-1.4}^{+3.0}\right) \times 10^{-14}$ & 0.54 & 4271 \\
${ }^{76} \mathrm{Ge}($ Cent. $)$ & $(7.3 \pm 0.6) \times 10^{-14}$ & 0.73 & 2039 \\
${ }^{8} \mathrm{Sa}($ Cent. $)$ & $\left(1.7_{-0.3}^{+0.4}\right) \times 10^{-13}$ & 1.70 & 2995 \\
${ }^{100} \mathrm{Mo}$ (Cent. $\left.\mathrm{Av}\right)^{\ddagger}$ & $(5.0 \pm 0.15) \times 10^{-13}$ & 5.0 & 3034 \\
${ }^{116} \mathrm{Cd}($ Cent. $A v)$ & $\left(1.3_{-0.3}^{+0.7}\right) \times 10^{-13}$ & 1.3 & 2802 \\
${ }^{130} \mathrm{Ta}($ Cent. $)$ & $(4.2 \pm 0.5) \times 10^{-13}$ & 4.3 & 2533 \\
${ }^{136} \mathrm{Xe}$ (Cent. $)$ & $(2.8 \pm 0.4) \times 10^{-14}$ & 0.28 & 2479 \\
${ }^{150} \mathrm{Nd}$ (Cent. $)$ & $\left(5.7_{-0.7}^{+1.0}\right) \times 10^{-12}$ & 57.0 & 3367 \\
\hline
\end{tabular}

$\dagger$ Cent. Centrifuge Separation.

$\ddagger$ Av. Atomic Vapor Laser Ionization Separation.

Table 2

Estimated half-lives corresponding to $\left\langle m_{\nu}\right\rangle=0.04 \mathrm{eV}$, and corresponding decay rates.

\begin{tabular}{|l|c|c|}
\hline \hline Isotope & $T_{1 / 2}^{0 \nu} y$ & $\lambda_{\beta \beta} y^{-1}$ \\
\hline${ }^{48} \mathrm{Ca}$ & $3.0 \times 10^{27}$ & $2.3 \times 10^{-28}$ \\
${ }^{76} \mathrm{Ge}$ & $2.3 \times 10^{27}$ & $3.0 \times 10^{-28}$ \\
${ }^{82} \mathrm{Se}$ & $9.4 \times 10^{26}$ & $7.4 \times 10^{-28}$ \\
${ }^{100} \mathrm{Mo}$ & $3.3 \times 10^{26}$ & $2.1 \times 10^{-27}$ \\
${ }^{116} \mathrm{Cd}$ & $1.3 \times 10^{27}$ & $5.3 \times 10^{-28}$ \\
${ }^{130} \mathrm{Te}$ & $3.9 \times 10^{26}$ & $1.8 \times 10^{-27}$ \\
${ }^{136} \mathrm{Xe}$ & $5.8 \times 10^{27}$ & $1.2 \times 10^{-28}$ \\
${ }^{150} \mathrm{Nd}$ & $2.9 \times 10^{25}$ & $2.4 \times 10^{-26}$ \\
\hline
\end{tabular}

Table 3

Atoms of parent isotopes of the various sources of $0 \nu \beta \beta$-decay per kilogram and estimated decay rates for $\left\langle m_{\nu}\right\rangle \sim 0.04$.

\begin{tabular}{|l|l|c|c|l|}
\hline \hline Isotope: form & $\mathrm{N} \mathrm{kg}^{-1}$ & $\lambda_{\beta \beta}($ estimate $) y^{-1}$ & $N \lambda_{\beta \beta} \mathrm{kg}^{-1} \mathrm{y}^{-1}$ & Abundance \\
\hline${ }^{48} \mathrm{Ca}: \mathrm{CaWO}_{4}$ & $1.34 \times 10^{24}$ & $2.3 \times 10^{-28}$ & $3.1 \times 10^{-4}$ & $80 \%$ \\
${ }^{48} \mathrm{Ca}: \mathrm{CF}_{2}$ & $1.44 \times 10^{22}$ & $2.3 \times 10^{-28}$ & $3.3 \times 10^{-6}$ & $0.187 \%$ \\
${ }^{76} \mathrm{Ge}: \mathrm{Ge}$ & $6.81 \times 10^{24}$ & $3.0 \times 10^{-28}$ & $2.0 \times 10^{-3}$ & $86 \%$ \\
${ }^{8} \mathrm{Se}: \mathrm{SeO}_{2}$ & $4.61 \times 10^{24}$ & $7.4 \times 10^{-28}$ & $3.4 \times 10^{-3}$ & $85 \%$ \\
${ }^{100} \mathrm{Mo}: \mathrm{Mo}^{116} \mathrm{Cd}: \mathrm{CdWO}_{4}$ & $5.41 \times 10^{24}$ & $2.1 \times 10^{-27}$ & $1.1 \times 10^{-2}$ & $90 \%$ \\
${ }^{130} \mathrm{Te}: \mathrm{TeO}_{2}$ & $1.34 \times 10^{24}$ & $5.3 \times 10^{-28}$ & $7.1 \times 10^{-4}$ & $80 \%$ \\
${ }^{130} \mathrm{Te}: \mathrm{TeO}_{2}$ & $3.21 \times 10^{24}$ & $1.8 \times 10^{-27}$ & $2.3 \times 10^{-3}$ & $33.8 \%$ \\
${ }^{136} \mathrm{Xe}: \mathrm{Xe}^{150} \mathrm{Nd}: \mathrm{Nd}_{2} \mathrm{O}_{3}$ & $1.54 \times 10^{24}$ & $1.2 \times 10^{-27}$ & $5.8 \times 10^{-3}$ & $85 \%$ \\
${ }^{15}$ & $1.47 \times 10^{24}$ & $2.4 \times 10^{-26}$ & $4.3 \times 10^{-4}$ & $80 \%$ \\
\hline
\end{tabular}

